\title{
Assistenza infermieristica e wound care nel paziente affetto da insufficienza renale cronica in trattamento dialitico
}

\author{
Stefano Mancin, Elena Alterchi, Silvia Finazzi, Salvatore Badalamenti \\ Dialysis clinic IRCCS Humanitas Research Hospital Rozzano (MI), Italia
}

\begin{abstract}
RIASSUNTO
Le persone affette da insufficienza renale cronica in trattamento dialitico, rappresentano un campione di popolazione ad alto rischio di sviluppo di patologie a carico della cute, in particolar modo lesioni secondarie all'uremia come xerosi e prurito, ulcere diabetiche e vascolari. Le cause sono riconducibili principalmente ai cambiamenti metabolici indotti dalla patologia quali complicanze uremiche, acidosi metabolica e alterazione del metabolismo calcio-fosforo e la concomitante presenza di patologie croniche, in primis diabete, arteriopatie e patologie cardiovascolari. Nel nostro centro dialisi tale problema incide notevolmente sulla qualità di vita della nostra utenza, rappresentando un problema serio e invalidante. Questo progetto di durata triennale 2016-2018, terminato con l'analisi del database nel 2019 ha portato a un miglioramento dell'assistenza infermieristica erogata presso il nostro ambulatorio, in termini di riduzione dell'incidenza di lesioni difficili pari al 46\% durante il triennio, implementazione delle conoscenze del personale coinvolto, utilizzo ottimale di presidi di medicazione avanzata e in ultimo una soddisfazione dei nostri utenti, relativamente al trattamento delle ferite, calcolata mediante questionario di gradimento, che ha mostrato una elevata partecipazione durante l'ultima indagine (98\%) e una soddisfazione per le cure prestate pari al $95 \%$.
\end{abstract}

\section{INTRODUZIONE}

Le patologie cutanee, rappresentano un problema serio e invalidante che ha un'alta incidenza nelle persone affette da insufficienza renale cronica in trattamento dialitico, ${ }^{1,2}$ poiché questa tipologia di paziente presenta un alto rischio

Corrispondenza: Stefano Mancin, Dialysis clinic IRCCS Humanitas Research Hospital Rozzano (MI), via Manzoni 56, 20089 Rozzano (MI), Italia.

E-mail: Stefano.mancin@humanitas.it

Parole chiave: Ulcere vascolari; ulcere del piede diabetico; wound care; dialisi; ferite traumatiche.

Conflitto di interessi: Gli autori dichiarano l'assenza di conflitto di interessi.

Disponibilità di dati e materiali: I dati analizzati in questo articolo sono disponibili su richiesta.

Approvazione etica e consenso alla partecipazione: Non applicabile.

Ricevuto per la pubblicazione: 24 dicembre 2020.

Accettato per la pubblicazione: 5 febbraio 2021.

This work is licensed under a Creative Commons Attribution NonCommercial 4.0 License (CC BY-NC 4.0).

${ }^{\circ}$ Copyright: the Author(s), 2021

Licensee PAGEPress, Italy

Italian Journal of Wound Care 2021; 5(1):73

doi:10.4081/ijwc.2021.73 di sviluppare lesioni difficili, a causa della patologia primaria e delle comorbilità associate.

L'eziologia di queste lesioni è riconducibile principalmente al diabete mellito ${ }^{3}$, all'arteriopatia, alle calcifilassi $i^{4,5}$ e alle manifestazioni secondarie della patologia uremica (xerosi, prurito, etc.).

La patologia è caratterizzata da un quadro clinico che evolve in un danno endoteliale, mediato da uno stato microinfiammatorio con elevati livelli di PCR, dallo stress ossidativo e carbonilico, dalla HB glicata elevata e dall'alterazione del metabolismo calcio-fosforo e del metabolismo lipidico. ${ }^{6}$ Inoltre, bisogna considerare che la maggior parte di questi pazienti è anziana, ad alto rischio di traumi accidentali (skin tears) che potrebbero evolvere in lesioni difficili (es. trauma in paziente diabetico).

Una gestione infermieristica precoce unita all'acquisizione di competenze, ${ }^{7}$ risulta essere un binomio vincente per la cura e trattamento di questa categoria di pazienti contribuendo a una riduzione delle complicanze e dei tempi di guarigione delle ferite.

\section{Osservazione e contesto}

Presso il nostro centro dialisi, annualmente afferiscono in media 110 pazienti, per la maggior parte persone anziane, difficilmente autosufficienti, ad alto rischio di sviluppare ulcere cutanee e con evidenti difficoltà a recarsi in ambulatori dedicati per effettuare visite di controllo ed esami diagnostici (anche a scopo preventivo).

Il campione è composto da una percentuale maschile pari al $61 \%$, con età media di 67 anni, la prevalenza di pazienti diabetici risulta essere pari al $42,5 \%$ e di pazienti 
affetti da arteriopatia pari al 32\%; i dati sono stati calcolati sulla media del triennio analizzato.

\section{Obiettivo}

Il progetto, di durata triennale (2016-18), ha lo scopo di migliorare l'assistenza infermieristica del paziente dializzato affetto da ulcera cutanea, di semplificare l'accesso ai servizi dedicati e di garantire una migliore qualità delle prestazioni erogate.

La prevenzione ricoprirà un ruolo primario poiché, in sinergia con l'educazione terapeutica, permetterà di riconoscere segni e sintomi di possibili complicanze nei pazienti ritenuti a rischio come per esempio soggetti diabetici e arteriopatici, anticipando il timing delle cure prestate.

\section{MATERIALI E METODI}

\section{Fase 1 (dicembre 2015)}

Nella prima fase, è stato analizzato il problema "lesioni difficili" in incontri di gruppo multidisciplinare. È stata introdotta una nuova figura, l'infermiere esperto in wound care, pensata come punto di riferimento per il paziente e per gli operatori sanitari e per migliorare la qualità delle cure erogate. Inoltre, sono stati organizzati eventi formativi atti a migliorare la conoscenza del personale medico-infermieristico sulla gestione delle lesioni difficili e sui presidi di medicazione avanzata.

\section{Fase 2 (dicembre 2015)}

Riprogrammazione del materiale presente in ambulatorio con l'introduzione di presidi di medicazioni avanzate, sulla base delle caratteristiche delle lesioni dei pazienti e in conformità con le più moderne linee guida. ${ }^{8}$

Inoltre, è stato istituito un archivio che raccolga i dati delle lesioni difficili, catalogati in base alla tipologia della lesione e suddivisi per anno d'insorgenza.

\section{Fase 3 (gennaio 2016)}

Creazione di una "scheda medicazioni," che si adatti alle caratteristiche del paziente cronico, al fine di garantire una migliore continuità delle cure. Introduzione di un questionario per valutare il grado di soddisfazione del paziente sull'assistenza ricevuta nella cura delle lesioni.

\section{Fase 4 (novembre 2016 - settembre 2018)}

Creazione di percorsi clinici dedicati alle due tipologie di lesioni più invalidanti nei pazienti: l'ulcera diabetica dell'arto inferiore e l'ulcera ischemica vascolare. Lo scopo di questi percorsi sarà facilitare le cure di queste tipologie di pazienti, garantendo un più rapido accesso agli ambulatori dedicati, velocizzando le cure e migliorando la prognosi del nostro paziente.

\section{Fase 5 (marzo 2017 - gennaio 2019)}

Inizio del programma educativo specifico e creazione di una brochure da consegnare ai pazienti ritenuti a rischio di sviluppare lesioni difficili allo scopo di prevenirne l'insorgenza, in particolar modo in pazienti diabetici, a rischio di ulcere ischemiche e a rischio di trauma accidentale (skin tears).

\section{Fase 6 (aprile 2018 - maggio 2018)}

Formazione del personale attraverso corso residenziale inerente la prevenzione e il trattamento di lesioni difficili del paziente con insufficienza renale.

\section{Fase 7 (2019) analisi dei dati e conclusione dello studio}

Verranno analizzati e confrontati i dati del triennio in studio al fine di valutare: i) Incidenza del numero di lesioni totale; ii) Incidenza del numero di lesioni diabetiche, arteriopatiche e skin tears; iii) Tempi di guarigione a 90 giorni; iv) Lesioni non guarite; v) Risultati questionari gradimento utenza; vi) Utilizzo presidi di medicazione avanzata.

\section{ANALISI DEI DATI}

\section{Lesioni difficili anno 2016}

Dei 115 pazienti afferenti presso il nostro centro, il $46 \%$ è diabetico ( $40 \mathrm{pz}$.) e il $34 \%$ presenta problemi vascolari (30 pz). Insorgenza di 68 lesioni difficili durante l'anno 2016 (Tabella 1).

Tabella 1. Lesioni difficili anno 2016.

\begin{tabular}{|c|c|c|c|c|}
\hline Anno 2016 & & & & \\
\hline $\begin{array}{l}\text { Pazienti } \\
\text { totali }\end{array}$ & & & $\begin{array}{c}\text { Pazienti con } \\
\text { problemi } \\
\text { vascolari }(\%)\end{array}$ & \\
\hline 115 & & & $30(34)$ & \\
\hline $\begin{array}{l}\text { Tipologia } \\
\text { lesioni }\end{array}$ & $\begin{array}{c}N^{\circ} \text { totale } \\
(\%)\end{array}$ & $\begin{array}{c}\text { Guarigione } \\
\text { a } 1 \text { mese } \\
(\%)\end{array}$ & $\begin{array}{c}\text { Guarigione } \\
\text { a } 3 \text { mesi } \\
(\%)\end{array}$ & $\begin{array}{c}\text { Non } \\
\text { guarite } \\
(\%)\end{array}$ \\
\hline Decubito & $6(9)$ & $5(83)$ & $1(17)$ & \\
\hline Chirurgiche & $3(4,5)$ & $1(33,3)$ & $2(66,7)$ & \\
\hline Traumatiche & $14(20,5)$ & $12(86)$ & $2(14)$ & \\
\hline Vascolari & $20(29)$ & & $16(80)$ & $4(20)$ \\
\hline Diabetiche & $25(37)$ & $9(36)$ & $7(28)$ & $6(24)$ \\
\hline Lesioni totali & 68 & & & \\
\hline
\end{tabular}


Lesioni da decubito: $9 \%$ delle lesioni totali (6 lesioni) localizzate per il 33\% (2 lesioni) alla zona sacro/glutei e per il 67\% (4 lesioni) agli arti inferiori; i) Guarigione ad 1 mese $83 \%$, (5 lesioni); ii) Guarigione a 3 mesi $17 \%$ (1 lesione).

Lesioni chirurgiche: $4,5 \%$ delle lesioni totali (3 lesioni) localizzate all'addome per il 33\% (1 lesione) e agli arti inferiori per il 67\% (2 lesioni): i) Guarigione ad 1 mese 33,3\%, (1 lesione); ii) Guarigione a 3 mesi $66,7 \%$ (2 lesioni).

Lesioni traumatiche: $20,5 \%$ delle lesioni totali (14 lesioni) localizzate per il 50\% (7 lesioni) agli arti superiori e per il 50\% agli arti inferiori (7 lesioni): i) Guarigione ad 1 mese 86\% (12 lesioni); ii) Guarigione a 3 mesi 14\% (2 lesioni).

Lesioni vascolari: $29 \%$ delle lesioni totali (20 lesioni), localizzate agli arti inferiori con origine arteriosa per il 95\% (19 lesioni) e con origine venosa per il 5\% (1 lesione): i) Guarigione a 3 mesi 80\% (16 lesioni); ii) Lesioni non guarite $20 \%$ (4 lesioni).

Lesioni diabetiche: $37 \%$ delle lesioni totali (25 lesioni). Di cui 40\% neuropatiche (10 lesioni), 48\% ischemiche (12 lesioni), 12\% miste (3 lesioni): i) Guarigione ad 1 mese 36\% (9 lesioni); ii) Guarigione a 3 mesi 28\% (7 lesioni); iii) Lesioni non guarite 24\% (6 lesioni).

\section{Lesioni difficili anno 2017}

Dei 110 pazienti afferenti presso in nostro centro, il $44 \%$ è diabetico (40 pz) e il $33 \%$ presenta problemi vascolari. Insorgenza di 39 lesioni difficili durante l'anno 2017 (Tabella 2).

Tabella 2. Lesioni difficili anno 2017.

\begin{tabular}{|c|c|c|c|c|}
\hline \multicolumn{5}{|l|}{ Anno 2017} \\
\hline $\begin{array}{l}\text { Pazienti } \\
\text { totali }\end{array}$ & \multicolumn{2}{|c|}{$\begin{array}{c}\text { Pazienti } \\
\text { diabetici } \\
(\%)\end{array}$} & \multicolumn{2}{|l|}{$\begin{array}{c}\text { Pazienti con } \\
\text { problemi } \\
\text { vascolari }(\%)\end{array}$} \\
\hline 110 & \multicolumn{2}{|c|}{$40(44)$} & \multicolumn{2}{|l|}{$30(33)$} \\
\hline $\begin{array}{l}\text { Tipologia } \\
\text { lesioni }\end{array}$ & $\begin{array}{c}N^{\circ} \text { totale } \\
(\%)\end{array}$ & $\begin{array}{c}\text { Guarigione } \\
\text { a } 1 \text { mese } \\
(\%)\end{array}$ & $\begin{array}{c}\text { Guarigione } \\
\text { a } 3 \text { mesi } \\
(\%)\end{array}$ & $\begin{array}{c}\text { Non } \\
\text { guarite } \\
(\%)\end{array}$ \\
\hline Decubito & $3(8)$ & $1(33,3)$ & & $2(66,7)$ \\
\hline Chirurgiche & $1(3)$ & $1(100)$ & & \\
\hline Traumatiche & $10(25,5)$ & $8(80)$ & $2(20)$ & \\
\hline Vascolari & $12(30,5)$ & & $8(66)$ & $2(17)$ \\
\hline Diabetiche & $13(33)$ & $5(61)$ & $1(8)$ & $2(15,5)$ \\
\hline Lesioni totali & 39 & & & \\
\hline
\end{tabular}

Lesioni da decubito: $8 \%$ delle lesioni totali (3 lesioni) localizzate per il 33\% (1 lesione) alla zona sacro/glutei e per il 67\% (2 lesioni) agli arti inferiori: i) Guarigione ad 1 mese $33 \%$ (1 lesione); ii) Non guarigione 66\% (2 lesioni).

Lesioni chirurgiche: 3\% delle lesioni totali (1 lesione) localizzata agli arti superiori: i) Guarigione a 1 mese $100 \%$.

Lesioni traumatiche: $25,5 \%$ delle lesioni totali (10 lesioni) localizzate per il 50\% agli arti superiori (5 lesioni), per il $30 \%$ agli arti inferiori (3 lesioni), per il 20\% al cranio (2 lesioni): i) Guarigione ad 1 mese 80\% (8 lesioni); ii) Guarigione a 3 mesi 20\% (2 lesioni).

Lesioni vascolari: $30,5 \%$ delle lesioni totali (12 lesioni), localizzate agli arti inferiori $100 \%$ di origine arteriosa: i) Guarigione a 3 mesi $66 \%$ (8 lesioni); ii) Non guarite 17\% (2 lesioni).

Lesioni diabetiche: $33 \%$ delle lesioni totali (13 lesioni): $38 \%$ neuropatiche (5 lesioni), $31 \%$ ischemiche (4 lesioni), $31 \%$ miste (4 lesioni): i) Guarigione a 1 mese $61 \%$ (5 lesioni); ii) Guarigione a 3 mesi $8 \%$ (1 lesione); iii) Lesioni non guarite 15,5\% (2 lesioni).

\section{Lesioni difficili anno 2018}

Dei 105 pazienti afferenti presso in nostro centro, il $38 \%$ è diabetico (40pz) e il $30 \%$ presenta problemi vascolari (32pz.). Insorgenza di 37 lesioni difficili durante l'anno 2018 (Tabella 3)

Lesioni da decubito: 11\% delle lesioni totali (4 lesioni) localizzate per il $75 \%$ alla regione sacrale (3 lesioni)

Tabella 3. Lesioni difficili anno 2018.

\begin{tabular}{|c|c|c|c|c|}
\hline \multicolumn{5}{|l|}{ Anno 2018} \\
\hline \multirow{2}{*}{$\begin{array}{l}\begin{array}{l}\text { Pazienti } \\
\text { totali }\end{array} \\
105\end{array}$} & \multicolumn{2}{|c|}{$\begin{array}{c}\text { Pazienti } \\
\text { diabetici } \\
(\%)\end{array}$} & \multirow[t]{2}{*}{$\begin{array}{c}\text { Pazienti con } \\
\text { problemi } \\
\text { vascolari }(\%)\end{array}$} & \multirow[b]{3}{*}{$\begin{array}{c}\text { Non } \\
\text { guarite } \\
(\%)\end{array}$} \\
\hline & $38(40)$ & $30(32)$ & & \\
\hline $\begin{array}{l}\text { Tipologia } \\
\text { lesioni }\end{array}$ & $\begin{array}{c}N^{\circ} \text { totale } \\
(\%)\end{array}$ & $\begin{array}{c}\text { Guarigione } \\
\text { a } 1 \text { mese } \\
(\%)\end{array}$ & $\begin{array}{c}\text { Guarigione } \\
\text { a } 3 \text { mesi } \\
(\%)\end{array}$ & \\
\hline Decubito & $4(11)$ & $2(50)$ & & $1(25)$ \\
\hline Chirurgiche & $1(33)$ & & $1(100)$ & \\
\hline Traumatiche & $9(24)$ & & $9(100)$ & \\
\hline Vascolari & $11(30)$ & $5(45)$ & $1(9)$ & $4(37)$ \\
\hline Diabetiche & $12(32)$ & & $11(92)$ & $1(8)$ \\
\hline Lesioni totali & 37 & & & \\
\hline
\end{tabular}


e per il $25 \%$ agli arti inferiori (1 lesione): i) Guarigione: a 1 mese 50\% (2 lesioni); ii) Lesioni non guarite $25 \%$ ( 1 lesione).

Lesioni chirurgiche: $3 \%$ delle lesioni totali (1 lesione) localizzata agli arti superiori. Guarigione a 3 mesi $100 \%$ (1 lesione).

Lesioni traumatiche: $24 \%$ delle lesioni totali (9 lesioni). Per il 55\% localizzate agli arti superiori (5 lesioni), per il 12\% allo sterno (1 lesione), per il 33\% agli arti inferiori (3 lesioni). Guarigione a 3 mesi 100\% (9 lesioni).

Lesioni vascolari: $30 \%$ delle lesioni totali (11 lesioni) di origine arteriosa. Localizzazione: arti inferiori (gamba) per il 45\% (5 lesioni) e agli arti inferiori (piede) per il 55\% (6 lesioni). i) Guarigione: ad 1 mese $45 \%$ (5 lesioni), ii) Guarigione a 3 mesi $9 \%$ (1 lesione), iii) Non guarigione $37 \%$ (4 lesioni).

Lesioni diabetiche: $32 \%$ delle lesioni totali (12 lesioni). Localizzazione: arti inferiori (piedi) per l' $83 \%$ (10 lesioni), arti inferiori (gamba) per 1'8,5\% (1 lesione) ed arti superiori per 1'8,5\% (1 lesione). i) Guarigione a 3 mesi 92\% (11 lesioni); ii) Non guarigione 8\% (1 lesione)

\section{RISULTATI}

Le lesioni maggiormente presenti nella nostra popolazione di pazienti è rappresentata dalle lesioni diabetiche $\mathrm{e}$, seguita dalle lesioni vascolari di origine arteriosa e dalle lesioni traumatiche, le lesioni da decubito e chirurgiche rappresentano una minoranza delle lesioni totali trattate.

Durante il triennio, è stata raggiunta, nel nostro centro, una riduzione del numero di lesioni difficili calcolando l'incidenza totale pari al $46 \%$, rispettivamente 68 casi nel 2016 a 37 casi nel 2018.

Analizzando le singole tipologie di lesioni, è stata raggiunta una riduzione delle ferite complessivamente del: i) $21 \%$ per le lesioni vascolari, rispettivamente 20 nel 2016 e 11 nel 2018, ii) $26 \%$ per le lesioni diabetiche, rispettivamente 25 nel 2016 e 12 nel 2018, iii) $17 \%$ per le lesioni traumatiche (skin tears), rispettivamente 14 nel 2016 e 9 nel 2018.

È stata valutata la guarigione a 90 giorni nelle tre tipologie di lesioni maggiormente presenti nel nostro campione con i seguenti risultati: i) Le lesioni diabetiche mostrano un aumento della guarigione a 3 mesi nel 2018 con il $92 \%$ di guarigione rispetto al 2016 in cui era pari al $64 \%$; ii) Le lesioni traumatiche hanno mostrato un trend di guarigione pressoché identico nel triennio raggiungendo sempre a 90 giorni una guarigione totale delle ferite trattate; iii) Le lesioni vascolari mostrano un incremento di guarigione che passa dal 16\% nel 2016 al 54\% nel 2018

Infine, analizzando $i$ dati delle lesioni non guarite, $i$ dati mostrano: i) Una riduzione per le lesioni diabetiche, dal $24 \%$ del 2016 all' $8 \%$ del 2018 ; ii) Le lesioni vascolari, mostrano un trend negativo, poiché passano dal $20 \%$ del 2016 , al $37 \%$ del 2018 , dato che potrebbe essere motivato dal fatto che con la riduzione del numero di lesioni vascolari nel triennio, le ferite trattate nell'ultimo anno risultano più complesse e necessitano di tempi di guarigione più lunghi.

\section{Risultati questionari di gradimento del servizio}

Annualmente, è stato distribuito un questionario per valutare la soddisfazione sul servizio di prevenzione trattamento delle lesioni difficili offerto ai nostri utenti. In particolar modo, sono state valutate le seguenti tematiche: assistenza del team multidisciplinare (1), qualità delle cure erogate (2), accesso agli ambulatori dedicati e alle visite specialistiche (3), educazione al paziente (4) e consapevolezza del "problema" lesioni difficili (5), per ogni item considerato venivano sottoposte 4 domande con una scala di gradimento da 1 a 10 per un totale di 20 risposte.

L'outcome considerato era il raggiungimento di una percentuale di gradimento $\geq$ del $85 \%$, con una partecipazione minima $\geq$ all' $80 \%$. I dati vengono riassunti nella Tabella 4 .

\section{Risultati utilizzo presidi di medicazione avanzata}

E' stato analizzato l'utilizzo da parte del team multidisciplinare nel triennio 2016-18 di tre presidi di medicazione avanzata più utilizzati: schiuma di poliuretano, Idrogel e Alginato. I dati nella Tabella 5 mostrano l'aumento di sen-

Tabella 4. Risultati questionario di gradimento utenza.

\begin{tabular}{lccc}
\hline Questionari gradimento utenza & & & \\
\hline Anno & $\mathbf{2 0 1 6}$ & $\mathbf{2 0 1 7}$ & $\mathbf{2 0 1 8}$ \\
\hline Partecipazione (No.) & $101(88 \%)$ & $103(94 \%)$ & $102(98 \%)$ \\
\hline Gradimento utenza totale (\%) & 88 & 94 & 95 \\
\hline Item 1 & 90 & 94 & 98 \\
\hline Item 2 & 98 & 98 & 98 \\
\hline Item 3 & 80 & 88 & 88 \\
\hline Item 4 & 90 & 98 & 98 \\
\hline Item 5 & 82 & 90 & 94 \\
\hline
\end{tabular}

Tabella 5. Utilizzo annuale presidi di medicazione avanzata.

\begin{tabular}{|cccc}
\hline Anno & $\begin{array}{c}\text { Schiuma di } \\
\text { poliuretano }\end{array}$ & Idrogel & Alginato \\
\hline 2016 & 174 & 59 & 10 \\
\hline 2017 & 220 & 59 & 18 \\
\hline 2018 & 312 & 78 & 38 \\
\hline
\end{tabular}


sibilità del personale nel privilegiare presidi di medicazione avanzata, frutto delle conoscenze apprese durante i corsi di aggiornamento tenuti nel triennio 2016-18.

\section{CONCLUSIONI}

Il presente progetto, ha affrontato un tema rilevante nella nostra tipologia di utenza, mostrando risultati significativi, frutto del miglioramento dell'assistenza erogata nel trattamento delle lesioni difficili.

I punti chiave per ottenere questi risultati sono stati: una maggiore sensibilizzazione sia del team, sia del paziente riguardo alla percezione di questo problema, un adeguata formazione degli operatori che ha portato a un miglioramento dell'assistenza erogata e alla corretta gestione e utilizzo di presidi di medicazione avanzata e infine l'introduzione dell'infermiere esperto in wound care come punto di riferimento all'interno del team.

Bisogna inoltre considerare il ruolo dell'educazione terapeutica, effettuata attraverso colloqui con paziente e familiari e supportata con brochure, che ha giocato un ruolo fondamentale nella prevenzione, in particolar modo verso le skin tears, ovvero tutte quelle lesioni traumatiche che possono evolvere in situazioni di estrema gravità nei pazienti diabetici e/o vasculopatici e in cui la prevenzione ricopre un ruolo di primaria importanza in modo da evitare il trauma e quindi la l'insorgenza della ferita.

In conclusione, si può affermare come il presente progetto abbia raggiunto risultati decisamente positivi e abbia implementato 1 qualità dell'assistenza offerta, migliorando la qualità di vita dei pazienti con lesioni difficili che afferiscono presso il nostro ambulatorio.

\section{BIBLIOGRAFIA}

1. Raspovic KM, Ahn J, La Fontaine J, et al. End-stage renal disease negatively affects physical quality of life in patients with diabetic foot complications. Int J Low Extrem Wounds 2017;16:135-42.

2. Soleymanian T, Kokabeh Z, Ramaghi R, et al. Clinical outcomes and quality of life in hemodialysis diabetic patients versus non-diabetics, J Nephropathol 2017;6:81-9.

3. Rizzo R, Mancini E, Santoro A. Il coinvolgimento cutaneo nelle patologie metaboliche associate a malattia renale cronica. G Ital Nefrol 2014;31.

4. Savoia F, Gaddoni G, Patrizi A. Calcifilassi in pazienti dializzati, una malattia grave poco rispondente alle terapie: report di 4 casi clinici. G Ital Dermatol Venereol 2013;148:531-6

5. Piantanida M, Plastino G, Valente V, e tal. Calcifilassi: approccio multidisciplinare. G Ital Nefrol 2009;4:530-3.

6. Rahnama-Moghadam S, Motazedi T, Krejci-Manwaring J. Hemodialysis patient with finger ulcerations. J Fam Pract 2017;66:107-10.

7. Andreozzi GM, Antignani P, Arosio E. Modello di percorso diagnostico e terapeutico per l'arteriopatia obliterante periferica. Minerva Cardioangiol 2012;60:1-35.

8. Bakker K, Apelqvist J, Lipsky BA, et al. The 2015 IWGDF guidance documents on prevention and management of foot problems in diabetes: development of an evidence-based global consensus. Diabetes Metab Res Rev 2016;32:2-6. 